
\title{
R Reserach S Suare \\ Long non-coding RNA landscapes in benign and malignant thyroid neoplasms of distinct histological subtypes
}

Valentina Yakushina ( $\square$ vdyakushina@gmail.com )

Research Centre of Medical Genetics https://orcid.org/0000-0001-5236-9297

Alexander Lavrov

Research Centre for Medical Genetics

\section{Research article}

Keywords: long non-coding RNA, SLC26A4-AS1, TNRC6C-AS1, CRNDE, RMST, follicular thyroid carcinomas, follicular adenoma, papillary thyroid carcinoma, anaplastic thyroid carcinoma

Posted Date: December 16th, 2019

DOI: https://doi.org/10.21203/rs.2.18943/v1

License: (c) (i) This work is licensed under a Creative Commons Attribution 4.0 International License.

Read Full License 


\section{Abstract}

Background: The main types of thyroid neoplasms, follicular adenoma (FA), follicular thyroid carcinoma (FTC), classical and follicular variants of papillary carcinoma (cIPTC and fvPTC), anaplastic thyroid carcinoma (ATC), are differ in the prognosis, rate of progression and metastatic behavior. It can be supposed that there are specific patterns of IncRNAs involved in the development of clinical and morphological features. The IncRNA landscapes within distinct benign and malignant histological variants of thyroid neoplasm are unknown.

Methods: Comprehensive set of Microarray and RNA-Seq datasets was analyzed for the expression of IncRNAs in FA, FTC, fvPTC, cIPTC and ATC. The potential biological functions were evaluated via coexpression and enrichment analysis.

Results and conclusion: Abberant expression of IncRNA in FA, FTC, fVPTC, CIPTC and ATC was established. The IncRNAs common for benign and malignant neoplasms, specific for papillary carcinomas, specific for CIPTC, fVPTC and ATC are determined. The determined common and specific IncRNAs are found to be putatively involved into L1CAM interactions; processing of capped introncontaining pre-mRNA; Tryptophan metabolism; PCP/CE pathway and Beta-catenin independent WNT signaling; extracellular matrix organization and cell cycle and mitotic. The patterns of IncRNA expression in FA and FTC are appeared to be similar with no genes significantly differentially expressed within these subtypes. Previously known oncogenic and supressor IncRNAs (NR2F1-AS1, LINC00511, SLC26A4-AS1, CRNDE, LINC01116, RMST) are found aberrantly expressed in thyroid carcinomas. The findings enhance the understanding of IncRNA landscape in thyroid neoplasms and its role in thyroid cancer progression.

\section{Background}

The most common types of thyroid cancer are papillary carcinomas (PTC) and follicular carcinomas (FTC). PTC and FTC account for about $70-80 \%$ and $10-15 \%$ of all thyroid cancers, respectively. Both PTC and FTC are differentiated carcinomas, but the mutational landscape and biological behavior, such as typical localization of metastasis (lymph nodes in the neck for PTC, distant organs, particularly, lungs and bones for FTC) and prognostic clinical markers are distinct [1]. Within PTC, several variants can be distinguished, where classical (CIPTC) and follicular variants (fVPTC) are the most common. The fvPTC is composed of neoplastic follicles rather than papillae, but with follicular cells showing nuclear features characteristic of PTC, overall it has an indolent behavior. The benign counterpart of follicular carcinoma is follicular adenoma, and it is often challenging to differentiate them by cytology. Follicular adenomas (FA), FTC and fvPTC compose follicular-pattern thyroid tumors, sharing common mutational prevalence and clinical features [1, 2]. Anaplastic thyroid cancer (ATC) is the most advanced and aggressive thyroid cancer and the least likely to respond to treatment $[3,4]$.

The evidence of important role of long non-coding RNA (IncRNA) in tumor suppression, cancer progression, invasion and metastatic potential, and its prognostic and therapeutic value are increasing 
[5]. LncRNAs are RNA molecules of more than 200 nucleotides, which typically do not have functional open reading frame (however, bifunctional RNAs were discovered which function as both, protein-coding and non-coding). Many IncRNA genes have two or more exons and display 5'-capping, poly-adenylation and alternative splicing. Functions of IncRNA are implemented through different ways: recruiting transcription factors, chromatin organizers, or chromatin modifiers, forming a DNA-RNA triplex anchoring effector proteins to the gene promoter, decoying miRNAs and proteins, or interfering with protein post-translational modification $[5,6,7,8]$. Relative to the coding genes, IncRNA can be classified into intergenic (lincRNA); antisense (on the opposite strand of protein-coding locus); sense intronic or overlapping (on the same strand, transcript in introns of a coding gene, or contains a coding gene in its intron); retained intron (an alternatively spliced transcript containing intronic sequence); bidirectional (originates from the promoter region of a protein-coding gene, with transcription proceeding in the opposite direction on the other strand); 3-prime overlapping (overlap the 3'UTR of a protein-coding locus on the same strand). Today, the number of annotated IncRNA genes reached 14720 , according to Ensembl version 93 [9].

In thyroid cancer several IncRNAs were shown to have pathogenic and predictive role, including BANCR, FALEC, CNALPTC1, PVT1, NAMA, PTCSC1, PTCSC2, PTCSC3, TNRC6C-AS1 and others [10-21]. However, all of the studies considered only PTC and mostly none of the previous work took into account the difference between CIPTC and fvPTC. There are no published studies describing landscapes of IncRNA in ATC, FTC and FA. Nevertheless, IncRNAs differently expressed in ATC could reflect anaplastic features and be strong prognostic factors. As morphology and behavior of FTC differ from PTC it can be proposed that the landscape of IncRNAs in FTC would be different from that of PTC. Investigation of IncRNAs common and specific for FA and FTC is important in understanding their relations and revealing differential diagnostic markers.

This study aimed to find out IncRNAs specific and common for main types of thyroid neoplasms (FA, FTC, fVPTC, CIPTC and ATC). The expression data from microarray technology (8 datasets) and RNA-Seq technology (PRJEB11591 dataset and TCGA transcriptome data) were analyzed.

\section{Materials \& Methods}

\section{Microarray datasets}

Microarray datasets from Affymetrix Human Genome U133 Plus 2.0 Array (Platform GPL570) were selected from GEO. The following datasets were included: GSE3467, GSE60542, GSE35570, GSE76039, GSE53157, GSE33630, GSE65144, GSE29265. A total of 107 samples of normal tissue and 32 fvPTC, 48 cIPTC, 49 ATC were analyzed.

CEL files were downloaded and normalization was performed using gcrma $\mathrm{R}$ package. Microarray probes were annotated with Ensembl version 93 using biomaRt package [22].

\section{RNA-Seq datasets}


RNA-Seq dataset PRJEB11591 of Yoo SK et al. [23] was selected from EBI European Nucleotide Archive database (https://www.ebi.ac.uk/ena/data/view/PRJEB11591). PRJEB11591 is the most comprehensive available RNA-Seq dataset containing benign and malignant thyroid neoplasms (FA, FTC, fvPTC, CIPTC). PRJEB11591 samples included 81 normal thyroid tissues (NT), 26 FA, 30 FTC, 48 fvPTC and 77 clPTC.

FASTQ files were downloaded, alignment was performed by hisat2 [24]. Counts were calculated using featureCounts (Rsubread package) with annotation by Ensembl version 93 and Ensemble gene ID as grouping attribute [25]. Genes with low counts (less than 2 count in number of samples exceeding the size of lowest sample group) were filtered out, TMM normalization (edgeR package) and voom method of limma R package were applied.

In TCGA transcriptomic data 58 NT, 356 clPTC and 101 fvPTC were selecte. Samples of metastases, and other minor histological subtypes were excluded. Raw counts (HTSeq - Counts Workflow Type, briefly, STAR 2-pass alignment followed by gene expression count assessment with HTSeq) were downloaded from Genomic Data Commons Data Portal (GDC, https://portal.gdc.cancer.gov/). Genes with low counts (les then 1 count in number of samples exceeding the size of lowest sample group) were filtered out, followed by TMM normalization (edgeR package) and voom analyses of limma [26].

\section{Selection of IncRNA genes}

Protein coding genes and genes attributed to Havana biotypes not related to IncRNA were filtered out of count matrices. Genes of the following Havana biotypes were included in the analyzes: lincRNA, antisense, 3-prime overlapping ncRNA, bidirectional promoter IncRNA, misc RNA, processed transcript, sense intronic, sense overlapping.

\section{Statistical analysis}

To identify differentially expressed IncRNAs, linear modelling using Limma package was performed [27]. Genes with FDR adjusted P-value $\leq 0.01$ and fold change $(F C) \geq 2.0$ were considered being differentially expressed. Hierarchical clustering heatmap analysis of differentially expressed genes was performed using coolmap of limma.

\section{Validation}

For cIPTC and fvPTC, sets of genes found significantly differentially expressed at the previous step on Microarray, RNA-Seq PRJEB11591, and RNA-Seq TCGA datasets were processed with intersection. Genes found in all three datasets, and genes found in both RNA-Seq dataset but absent in microarray probes were considered as validated.

\section{Evaluation of potential biological functions}

To identify genes positively and negatively coexpressed with the differently expressed IncRNA pairwise Pearson correlation between the IncRNA and all the genes was calculated. Genes with an absolute $r \geq 0.7$ and a significant correlation (P-value $<0.05$ ) were considered to be coexpressed. Enrichment of Gene Ontology (GO) Biological Process (2018), GO Molecular Function (2018), Kyoto Encyclopedia of Genes 
and Genomes (KEGG, 2016) and Reactome (2016) terms was analyzed using Enrichr [28, 29]. Terms with adjusted P-value from Fisher's exact test $\leq 0.05$ were considered significantly enriched.

\section{Results}

\section{LncRNAs differentially expressed in thyroid neoplasms}

Differential expression was evaluated in main histological subtypes of thyroid nodules: FA, FTC, fVPTC, CIPTC, ATC. Differential expression was estimated in each of histological subtypes of thyroid nodule compared to normal tissue. Further, the sets of differentially expressed genes were compared against the other histological types to find specific patterns of expression.

Expression of 3910 IncRNA genes in microarray dataset, 2587 - in RNA-Seq PRJEB11591 dataset and 3009 - in RNA-Seq TCGA datasets was analyzed. Each dataset was examined separately with

subsequent meta-analysis of robustly expressed genes via intersection of the results for CIPTC and fvPTC (for the rest subtypes the only one dataset was available). For PTC differential expression of IncRNAs was considered validated in silico if it was registered in all 3 datasets, or in 2 RNA-seq datasets if a particular IncRNA is not covered by the microarray probes.

The numbers of differentially expressed IncRNA are represented in Table 1. The full lists of discovered differentially expressed IncRNA are in the Additional files 1-3. Volcano plots representing distribution of fold change and adjusted p-values in studied histological subtypes are in the Additional file 4.

By in silico validation, 116 genes were confirmed as validated in cIPTC ( 45 genes found in all analyzed datasets, plus 71 genes without probes in microarray found in both RNA-Seq datasets; Fig. 1A), and 62 genes - in fvPTC (Fig. 1B) - robustly differentially expressed IncRNA.

Table 1. Number of IncRNAs differentially expressed in thyroid nodules compared to normal thyroid tissue

\begin{tabular}{|llll|}
\hline Histological type of the nodule & Microarray & RNA-Seq PRJEB11591 & RNA-Seq THCA \\
\hline FA & & 143 & \\
\hline FTC & & 213 & 174 \\
\hline fVPTC & 84 & 213 & 308 \\
\hline CIPTC & 137 & 401 & \\
\hline ATC & 330 & & \\
\hline
\end{tabular}

Clustering analyses was performed for microarray and PRJEB11591 datasets. It showed strong clustering of ATC, clustering of CIPTC and weak clustering of fVPTC. Surprisingly there was no clustering within the groups of FTC and FA (Fig. 2). 


\section{LncRNAs common for benign and malignant thyroid neoplasms}

There are LINC02555 and LINC02471 genes that are in Top 5 differently expressed IncRNAs in all studied thyroid neoplasms, including FA (Fig. 3, 4, Table 2). These IncRNA are validated in CIPTC and fvPTC, and are differentially expressed in papillary carcinomas compared to FA.

\section{LncRNAs common for differentiated thyroid carcinomas}

There are 32 IncRNAs differentially expressed in all studied histological subtypes of differentiated carcinomas (FTC, cIPTC, fvPTC) but not in FA (Fig. 3). Of them, 6 IncRNAs were validated and significantly differentially expressed in CIPTC and fvPTC compared to FA (Fig. 4, Table 2). None of the 32 IncRNA was differentially expressed in FTC compared to FA.

Table 2. IncRNA common for thyroid neoplasms

\begin{tabular}{|lllllll|}
\hline ENSG ID & HGNC symbol & Gene biotype & \multicolumn{3}{l}{ Log(FC) compared to NT } \\
\cline { 5 - 7 } & & & FA & FTC & fvPTC & cIPTC \\
\hline ENSG00000256268 & LINC02454 & lincRNA & - & 2.0 & 4.2 & 4.5 \\
\hline ENSG00000225342 & - & antisense & - & 1.7 & 4.4 & 3.1 \\
\hline ENSG00000250343 & STK32A-AS1 & antisense & - & 1.6 & 3.1 & 3.2 \\
\hline ENSG00000272384 & - & lincRNA & - & 1.1 & 1.9 & 2.5 \\
\hline ENSG00000233251 & - & antisense & - & -1.4 & -2.3 & -1.6 \\
\hline ENSG00000254489 & - & antisense & - & -1.8 & -3.2 & -3.9 \\
\hline ENSG00000260943 & LINC02555 & lincRNA & 2.7 & 4.8 & 7.8 & 5.0 \\
\hline ENSG00000223914 & LINC02471 & lincRNA & 2.0 & 4.2 & 7.2 & 6.5 \\
\hline $\begin{array}{l}\text { Differential expression of all genes is validated in clPTC and fvPTC. Expression in fvPTC and clPTC } \\
\text { differs significantly compared to FA. }\end{array}$ & & & & & \\
\hline
\end{tabular}

\section{LncRNA specific for papillary carcinomas}

There are 22 genes differentially expressed in both cIPTC and fvPTC, but not in follicular neoplasms (Fig. 3), validated and significantly differentially expressed compared to FA and FTC (Fig. 4, Table 3) InRNAs associated with papillary features in thyroid carcinomas. 
Table 3

LncRNA specific for papillary carcinomas

\begin{tabular}{|c|c|c|c|c|}
\hline \multirow[t]{2}{*}{ ENSG ID } & \multirow[t]{2}{*}{ HGNC symbol } & \multirow[t]{2}{*}{ Gene biotype } & \multicolumn{2}{|c|}{$\log (\mathrm{FC})$ compared to NT } \\
\hline & & & fvPTC & clPTC \\
\hline ENSG00000237463 & & antisense & 4.2 & 6.4 \\
\hline ENSG00000203585 & LINC02408 & lincRNA & 2.0 & 4.8 \\
\hline ENSG00000251002 & & antisense & 4.2 & 4.7 \\
\hline ENSG00000272482 & & lincRNA & 1.7 & 3.6 \\
\hline ENSG00000204282 & TNRC6C-AS1 & antisense & 2.4 & 3.4 \\
\hline ENSG00000197301 & & antisense & 2.4 & 3.4 \\
\hline ENSG00000267199 & & antisense & 1.7 & 3.1 \\
\hline ENSG00000235978 & & antisense & 2.2 & 2.5 \\
\hline ENSG00000230910 & & antisense & 2.0 & 2.2 \\
\hline ENSG00000257989 & & lincRNA & 2.5 & 2.2 \\
\hline ENSG00000224020 & MIR181A2HG & antisense & 1.7 & 1.8 \\
\hline ENSG00000272079 & & lincRNA & 2.1 & 1.7 \\
\hline ENSG00000272512 & & lincRNA & 1.0 & 1.6 \\
\hline ENSG00000237742 & & antisense & 1.9 & 1.4 \\
\hline ENSG00000255366 & & lincRNA & 1.1 & 1.3 \\
\hline ENSG00000265666 & RARA-AS1 & antisense & 1.4 & 1.0 \\
\hline ENSG00000204934 & ATP6V0E2-AS1 & antisense & -1.1 & -1.5 \\
\hline ENSG00000228559 & & lincRNA & -1.2 & -1.6 \\
\hline ENSG00000234899 & SOX9-AS1 & lincRNA & -1.2 & -1.7 \\
\hline ENSG00000228613 & & antisense & -2.2 & -2.6 \\
\hline ENSG00000267034 & & lincRNA & -2.6 & -2.9 \\
\hline ENSG00000261399 & & antisense & -2.0 & -3.3 \\
\hline
\end{tabular}


There are 20 IncRNAs aberrantly expressed in FTC, but not in other studied neoplasms, and significantly differentially expressed compared to PTC (Table 4). However, none of these IncRNAs was differentially expressed compared to FA.

Table 4. IncRNA specific for FTC

\begin{tabular}{|llll|}
\hline ENSG ID & HGNC symbol & Gene biotype & Log(FC) compared to NT \\
\hline ENSG00000281383 & - & lincRNA & 1.4 \\
\hline ENSG00000272732 & - & lincRNA & -1.0 \\
\hline ENSG00000224660 & SH3BP5-AS1 & antisense & -1.0 \\
\hline ENSG00000225855 & RUSC1-AS1 & antisense & -1.0 \\
\hline ENSG00000197989 & SNHG12 & antisense & -1.0 \\
\hline ENSG00000198221 & AFDN-DT & lincRNA & -1.1 \\
\hline ENSG00000248019 & FAM13A-AS1 & antisense & -1.1 \\
\hline ENSG00000273576 & - & lincRNA & -1.1 \\
\hline ENSG00000261087 & - & lincRNA & -1.1 \\
\hline ENSG00000271895 & - & antisense & -1.2 \\
\hline ENSG00000242282 & - & lincRNA & -1.2 \\
\hline ENSG00000272374 & - & lincRNA & -1.2 \\
\hline ENSG00000204584 & - & antisense & -1.3 \\
\hline ENSG00000262370 & - & lincRNA & -1.3 \\
\hline ENSG00000205959 & - & lincRNA & -1.3 \\
\hline ENSG00000285103 & - & bidirectional_promoter_IncRNA & -1.4 \\
\hline ENSG00000276007 & - & sense_intronic & -1.4 \\
\hline ENSG00000226419 & SLC16A1-AS1 & antisense & -1.5 \\
\hline ENSG00000257671 & KRT7-AS & antisense & -1.6 \\
\hline
\end{tabular}

The 32 genes were found being differentially expressed in CIPTC but not in other differentiated carcinomas and FA, validated, and significantly differentially expressed compared to fvPTC, FTC and FA IncRNA specific for clPTC (Fig. 3, 4, Table 5). 
Table 5

LncRNA specific for classical variant of papillary carcinoma

\begin{tabular}{|c|c|c|c|}
\hline ENSG ID & HGNC symbol & Gene biotype & Log(FC) compared to NT \\
\hline ENSG00000227036 & LINC00511 & lincRNA & 2.5 \\
\hline ENSG00000237187 & NR2F1-AS1 & antisense & 2.5 \\
\hline ENSG00000260604 & - & lincRNA & 2.2 \\
\hline ENSG00000262903 & - & antisense & 2.1 \\
\hline ENSG00000261101 & - & sense_overlapping & 1.9 \\
\hline ENSG00000274021 & - & antisense & 1.8 \\
\hline ENSG00000281406 & BLACAT1 & lincRNA & 1.5 \\
\hline ENSG00000245571 & FAM111A-DT & lincRNA & 1.3 \\
\hline ENSG00000253930 & TNFRSF10A-AS1 & antisense & 1.3 \\
\hline ENSG00000235609 & - & lincRNA & 1.2 \\
\hline ENSG00000237943 & PRKCQ-AS1 & lincRNA & -1.0 \\
\hline ENSG00000260572 & - & antisense & -1.0 \\
\hline ENSG00000204860 & FAM201A & antisense & -1.0 \\
\hline ENSG00000177640 & CASC2 & antisense & -1.1 \\
\hline ENSG00000259704 & - & sense_overlapping & -1.1 \\
\hline ENSG00000231769 & - & antisense & -1.2 \\
\hline ENSG00000231231 & LINC01423 & lincRNA & -1.2 \\
\hline ENSG00000272622 & - & lincRNA & -1.2 \\
\hline ENSG00000251602 & - & antisense & -1.3 \\
\hline ENSG00000231856 & - & antisense & -1.3 \\
\hline ENSG00000249249 & - & antisense & -1.3 \\
\hline ENSG00000205791 & LOH12CR2 & lincRNA & -1.3 \\
\hline ENSG00000232415 & ELN-AS1 & antisense & -1.5 \\
\hline ENSG00000262185 & - & sense_overlapping & -1.8 \\
\hline ENSG00000224885 & EIPR1-IT1 & sense_intronic & -1.9 \\
\hline ENSG00000256151 & ADGRD1-AS1 & lincRNA & -1.9 \\
\hline
\end{tabular}




\begin{tabular}{|llll|}
\hline ENSG ID & HGNC symbol & Gene biotype & Log(FC) compared to NT \\
\hline ENSG00000231107 & LINC01508 & lincRNA & -2.0 \\
\hline ENSG00000267128 & RNF157-AS1 & antisense & -2.0 \\
\hline ENSG00000229457 & LINC01789 & lincRNA & -2.0 \\
\hline ENSG00000249487 & LINC01586 & lincRNA & -2.7 \\
\hline ENSG00000224568 & LINC01886 & lincRNA & -2.7 \\
\hline ENSG00000233705 & SLC26A4-AS1 & antisense & -3.0 \\
\hline
\end{tabular}

Of 29 genes differently expressed in fVPTC but not in other differentiated carcinomas or FA (Fig. 3), only ENSG00000257647 gene is specific for fvPTC - validated and significantly differentially expressed in fvPTC compared to FA, FTC and clPTC.

\section{LncRNA specific for ATC}

ATC samples were available only in microarray dataset, which also included two variants of PTC. Out of 376 IncRNAs differentially expressed in ATC, 252 were not differentially expressed in other investigated histological subtypes, and 185 were significantly differentially expressed compared to CIPTC and fvPTC

- IncRNAs specific for ATC. Top 30 genes are represented in Table 6, the full list is in the Additional file 5. 
Table 6

Top 30 IncRNA specific for anaplastic carcinoma

\begin{tabular}{|c|c|c|c|}
\hline ENSG ID & HGNC symbol & Gene biotype & Log(FC) compared to NT \\
\hline ENSG00000272872 & - & sense_intronic & 3.5 \\
\hline ENSG00000244158 & - & antisense & 3.2 \\
\hline ENSG00000245694 & CRNDE & lincRNA & 3.2 \\
\hline ENSG00000240476 & LINC00973 & lincRNA & 3.1 \\
\hline ENSG00000282638 & - & lincRNA & 3.0 \\
\hline ENSG00000247134 & - & lincRNA & 3.0 \\
\hline ENSG00000254615 & - & lincRNA & 2.9 \\
\hline ENSG00000280018 & - & lincRNA & 2.8 \\
\hline ENSG00000233682 & - & antisense & -2.5 \\
\hline ENSG00000266904 & LINC00663 & lincRNA & -2.6 \\
\hline ENSG00000228506 & - & antisense & -2.6 \\
\hline ENSG00000275234 & - & antisense & -2.7 \\
\hline ENSG00000232229 & LINC00865 & lincRNA & -2.8 \\
\hline ENSG00000269609 & RPARP-AS1 & lincRNA & -2.9 \\
\hline ENSG00000270820 & - & antisense & -2.9 \\
\hline ENSG00000271474 & - & antisense & -3.0 \\
\hline ENSG00000284644 & - & antisense & -3.0 \\
\hline ENSG00000273015 & - & lincRNA & -3.1 \\
\hline ENSG00000260686 & - & sense_overlapping & -3.2 \\
\hline ENSG00000180769 & WDFY3-AS2 & antisense & -3.2 \\
\hline ENSG00000247400 & DNAJC3-DT & lincRNA & -3.2 \\
\hline ENSG00000271858 & - & antisense & -3.3 \\
\hline ENSG00000236155 & - & processed_transcript & -3.3 \\
\hline ENSG00000224078 & SNHG14 & antisense & -3.5 \\
\hline ENSG00000250073 & - & antisense & -3.8 \\
\hline ENSG00000203709 & MIR29B2CHG & lincRNA & -4.1 \\
\hline
\end{tabular}




\begin{tabular}{|llll|}
\hline ENSG ID & HGNC symbol & Gene biotype & Log(FC) compared to NT \\
\hline ENSG00000261183 & SPINT1-AS1 & antisense & -4.2 \\
\hline ENSG00000229891 & LINC01315 & lincRNA & -4.3 \\
\hline ENSG00000257151 & PWAR6 & lincRNA & -4.8 \\
\hline ENSG00000255794 & RMST & lincRNA & -5.7 \\
\hline
\end{tabular}
Potential biological functions of aberrantly expressed
IncRNAS

For IncRNAs common for studied histological subtypes and top 5 specific IncRNA in each subtype, enrichment of Gene Ontology (GO) biological processes, GO molecular functions, KEGG, and Reactome terms with coexpressed coding genes was established. The main related functions of the common IncRNAs is L1CAM interactions; IncRNA specific for FTC - processing of capped intron-containing premRNA; specific for papillary carcinomas - Tryptophan metabolism; specific for fvPTC - PCP/CE pathway and Beta-catenin independent WNT signaling; specific for clPTC - extracellular matrix organization; specific for ATC - cell cycle and mitotic (Fig. 5).

\section{Discussion}

Histological subtypes of follicular cell-derived thyroid carcinomas (FTC, PTC, ATC) significantly differ in their mutational landscapes and clinical characteristics. Although FTC and cIPTC both are differentiated carcinomas, FTC are characterized by follicular growth pattern and tend more often to spread metastases to distant organs, while cIPTC typically have papillary architecture and spread more often to lymph nodes in the neck. In FTC K/H/NRAS and PAX8/PPARG mutations are prevalent, whereas BRAF mutations and tyrosine kinase fusions prevail in cIPTC [1]. fvPTC are composed of neoplastic follicles rather than papillae, but with follicular cells showing nuclear features of PTC. The clinical characteristics of fvPTC are intermediate [30]. The mutational profile of fvPTC is more similar to FTC: prevalence of K/H/NRAS and PAX8/PPARG mutations. In the TCGA study, fvPTC were characterized as Ras-like tumors and their classification as papillary carcinomas was questioned [31]. Recently, reclassification of encapsulated fVPTC as "noninvasive follicular thyroid neoplasm with papillary-like nuclear features" (NIFTP) was proposed [2]. FA are thought to be benign counterpart of FTC and understanding the common and different molecular features of these neoplasms is important for the development of diagnostic and therapeutic strategy. ATC are thought to be an advanced stage of thyroid neoplasms, it is the most aggressive thyroid cancer and it is expected that there are specific molecular features, including IncRNA, associated with its aggressive behavior.

In this study, expression of IncRNA was evaluated in the main histological subtypes of thyroid neoplasm: FA, FTC, fvPTC, CIPTC and ATC. Datasets analyzed in the study (Microarray dataset of 8 independent experiments; RNA-Seq PRJEB11591 and RNA-Seq TCGA) allowed to perform robust in silico validation of 
the results for CIPTC and fvPTC, and to include representative set of FA, FTC and ATC samples. LncRNA landscapes in FA, FTC and ATC were analyzed for the first time.

LncRNAs general for thyroid neoplasms, specific for cIPTC, fvPTC, ATC and general for papillary carcinomas (associated with papillary features) were discovered. The highest number of differently expressed genes was in ATC (330 IncRNAs) followed by cIPTC (137), FTC and fvPTC that reflects the more advanced stage of ATC. The IncRNAs specific for ATC and probably associated with anaplastic features were discovered for the first time. None of IncRNAs specific for FTC is differentially expressed compared to FA. The only IncRNA ENSG00000257647 is specific for fvPTC that might be explained by its intermediate morphology combining features of both papillary and follicular carcinomas and debatable classification.

Aberrant expression of some IncRNAs previously found by Liyanarachchi S. et all. (2016) was confirmed in our study. Most of these IncRNAs occurred to be general for thyroid carcinomas [14]. Previously known promoters of cancer progression were found upregulated in thyroid carcinomas: NR2F1-AS1 and LINC00511 - in CIPTC; TNRC6C-AS1 - in CIPTC and fVPTC; CRNDE - in ATC. Known putative tumor suppressors were identified within downregulated IncRNAs: SLC26A4-AS1 - in CIPTC and ATC; RMST in ATC [32-38].

Putative biological process involving common and specific IncRNAs were established. LncRNAs common for all studied thyroid neoplasms might be involved in L1CAM interactions; common for follicular and classical variants of papillary carcinoma - in Tryptophan metabolism. Tryptophan degradation to kynurenine by the Indoleamine 2,3-Dioxygenase 1 (IDO1) is a well characterized immunosuppressive mechanism in cancer progression, including thyroid cancer [39].

Biological processes involving IncRNAs specific for FTC include processes that are associated with splicing (Processing of Capped Intron-Containing Pre-mRNA, mRNA Splicing, RNA processing). Accumulating evidence suggests that aberrant RNA splicing is a common and driving event in cancer development and progression. For instance, oncogenic Ras signaling via ERK and PI3-K/Akt pathways is described to regulate phosphorylation of splicing factors such as SRSF1, SRSF7, SPF45 and drive switching active and inactive states of tumor promoters and suppressors (MST1R, FAS, CD44, LBR, Casp$9, \mathrm{KLF} 6$, and others) via alternative splicing $[40,41]$.

LncRNA ENSG00000257647 specific for fvPTC appeared to be involved in WNT signaling, predominantly Beta-catenin independent WNT pathway (especially, planar cell polarity that modulates cytoskeleton rearrangements through the activation of the small GTPases RhoA and Rac and their downstream effectors Rock and JNK). WNT signaling is described to play a crucial role in thyroid carcinogenesis, several mechanisms of its deregulation were described, including inhibition of $\beta$-catenin degradation complex via its phosphorylation by RET/PTC and decrease of E-cadherin expression by MAPK/ERK pathway activated by BRAF mutations. RAS mutations are described to activate both, canonical and noncanonical Wnt pathways in thyroid carcinomas $[42,43]$. 
LncRNAs specific for clPTC are involved into extracellular matrix organization and collagen formation. Extracellular matrix (ECM) disorganization is known to play a pivotal role in cancer initiation and progression. There is emerging evidence of ECM remodeling induced by BRAF p.V600E in PTCs [44]. Notably, it was shown that extracellular matrix of PTCs driven by BRAF p.V600E (but not mutant HRAS) is enriched with stromal-derived fibrillar collagen and it facilitates cancer progression [45].

For IncRNAs specific for ATC there is a strong enrichment of cell cycle and mitotic pathways which possibly reflects involvement of these IncRNAs in the loss of differentiation and high proliferation rate characteristic for ATC.

\section{Conclusion}

LncRNAs common for thyroid neoplasms, common for carcinomas with papillary features, specific for CIPTC, fvPTC, FTC and ATC were discovered in the performed analyses of the most comprehensive dataset (combined of Microarray dataset and two RNA-Seq datasets). Similarity of IncRNA landscapes in FTC and FA was revealed. LncRNAs found to be specific for ATC are probably associated with anaplastic features and cancer progression.

\section{Declarations}

Ethics approval and consent to participate. Not applicable

Consent for publication. Not applicable

Availability of data and materials. The datasets used and analysed during the current study are available in the following repositories:

GEO (https://www.ncbi.nlm.nih.gov/geo/, GSE3467, GSE60542, GSE35570, GSE76039, GSE53157, GSE33630, GSE65144, GSE29265);

Genomic Data Commons Data Portal (https://portal.gdc.cancer.gov/);

EBI European Nucleotide Archive database (https://www.ebi.ac.uk/ena/data/view/PRJEB11591).

Competing interests. The authors declare that they have no competing interests

Funding. The research was carried out within the state assignment of Ministry of Science and Higher Education of the Russian Federation

Authors' contributions. VY performed bioinformatic and statistical analysis and wrote the manuscript. AL supervised the work. All authors read and approved the final manuscript.

Acknowledgements. Not applicable 


\section{References}

1. Acquaviva G, Visani M, Repaci A, Rhoden KJ, de Biase D, Pession A, et al. Molecular pathology of thyroid tumours of follicular cells: a review of genetic alterations and their clinicopathological relevance. Histopathology. 2018;72(1):6-31.

2. Nikiforov YE, Seethala RR, Tallini G, Baloch ZW, Basolo F, Thompson LD, et al. Nomenclature Revision for Encapsulated Follicular Variant of Papillary Thyroid Carcinoma. JAMA Oncol. 2016;2(8):10231029.

3. Cabanillas ME, Zafereo M, Gunn GB, Ferrarotto R. Anaplastic Thyroid Carcinoma: Treatment in the Age of Molecular Targeted Therapy. J Oncol Pract. 2016;12(6): 511-518.

4. Landa I, Ibrahimpasic T, Boucai L, Sinha R, Knauf JA, Shah RH, et al. Genomic and transcriptomic hallmarks of poorly differentiated and anaplastic thyroid cancers. J Clin Invest. 2016;126(3): 10521066.

5. Bure IV, Kuznetsova EB, Zaletaev DV. Long Noncoding RNAs and Their Role in Oncogenesis. Mol Biol. 2018;52(6):787-798.

6. Derrien T, Johnson R, Bussotti G, Tanzer A, Djebali S, Tilgner H, et al. The GENCODE v7 catalog of human long noncoding RNAs: analysis of their gene structure, evolution, and expression. Genome Res. 2012;22(9):1775-1789.

7. Fang Y, Fullwood MJ. Roles, Functions, and Mechanisms of Long Non-coding RNAs in Cancer. Genomics Proteomics Bioinformatics. 2016;14(1):42-54.

8. Li R, Zhu H, Luo Y. Understanding the Functions of Long Non-Coding RNAs through Their HigherOrder Structures. Int J Mol Sci. 2016;17(5):E702.

9. Zerbino DR, Achuthan P, Akanni W, Amode MR, Barrell D, Bhai J, et al. Ensembl 2018. Nucleic Acids Research. 2018;46(D1):D754-D761.

10. Yoon H, He H, Nagy R, Davuluri R, Suster S, Schoenberg D, et al. Identification of a novel noncoding RNA gene, NAMA, that is downregulated in papillary thyroid carcinoma with BRAF mutation and associated with growth arrest. Int J Cancer. 2007;121(4):767-775.

11. He H, Nagy R, Liyanarachchi S, Jiao H, Li W, Suster S, et al. A susceptibility locus for papillary thyroid carcinoma on chromosome 8q24. Cancer Res. 2009;69:625-631.

12. Jeong S, Lee J, Kim D, Seol MY, Lee WK, Jeong JJ, et al. Relationship of Focally Amplified Long Noncoding on Chromosome 1 (FAL1) IncRNA with E2F Transcription Factors in Thyroid Cancer. Med. 2016;95:e2592.

13. Liao T, Qu N, Shi RL, Guo K, Ma B, Cao YM, et al. BRAF-activated LncRNA functions as a tumor suppressor in papillary thyroid cancer. Oncotarget. 2016;8(1):238-247.

14. Liyanarachchi S, Li W, Yan P, Bundschuh R, Brock P, Senter L, et al. Genome-wide expression screening discloses long noncoding RNAs involved in thyroid carcinogenesis. J Clin Endocrinol Metab. 2016;101(11):4005-4013. 
15. Wang Q, Yang $H$, Wu L, Yao J, Meng X, Jiang H, et al. Identification of Specific Long Non-Coding RNA Expression: Profile and Analysis of Association with Clinicopathologic Characteristics and BRAF Mutation in Papillary Thyroid Cancer. Thyroid. 2016;26 (12):1719-1732.

16. Zheng $\mathrm{H}$, Wang $\mathrm{M}$, Jiang $\mathrm{L}$, Chu $\mathrm{H}, \mathrm{Hu} \mathrm{J}$, Ning J, et al. BRAF-Activated Long Noncoding RNA Modulates Papillary Thyroid Carcinoma Cell Proliferation through Regulating Thyroid Stimulating Hormone Receptor. Cancer Res Treat. 2016;48(2):698-707.

17. Zhou Q, Chen J, Feng J, Wang J. Long noncoding RNA PVT1 modulates thyroid cancer cell proliferation by recruiting EZH2 and regulating thyroid-stimulating hormone receptor (TSHR). Tumor Biol. 2016;37(3):3105-3113.

18. Muhanhali D, Zhai T, Jiang J, Ai Z, Zhu W, Ling Y. Long Non-coding Antisense RNA TNRC6C-AS1 Is Activated in Papillary Thyroid Cancer and Promotes Cancer Progression by Suppressing TNRC6C Expression. Front Endocrinol (Lausanne). 2018;9:360.

19. Hou S, Lin Q, Guan F, Lin C. LncRNA TNRC6C-AS1 regulates UNC5B in thyroid cancer to influence cell proliferation, migration, and invasion as a competing endogenous RNA of miR-129-5p. J Cell Biochem. 2018;119:8304-8316.

20. Chen C, Zhou L, Wang H, Chen J, Li W, Liu W, et al. Long noncoding RNA CNALPTC1 promotes cell proliferation and migration of papillary thyroid cancer via sponging miR-30 family. Am J Cancer Res. 2018;8(1):192-206.

21. Huang $\mathrm{H}$, Chen J, Ding C-M, Jin X, Jia Z-M, Peng J. LncRNA NR2F1-AS1 regulates hepatocellular carcinoma oxaliplatin resistance by targeting ABCC1 via miR-363. J Cell Mol Med. 2018;22:32383245.

22. Yoo SK, Lee S, Kim SJ, Jee HG, Kim BA, Cho H, et al. Comprehensive Analysis of the Transcriptional and Mutational Landscape of Follicular and Papillary Thyroid Cancers. PLoS Genet. 2016; 12(8):e1006239.

23. Durinck S, Spellman PT, Birney E, Huber W. Mapping identifiers for the integration of genomic datasets with the R/Bioconductor package biomaRt. Nat Protoc. 2009;4:1184-1191.

24. Kim D, Langmead B, Salzberg SL. HISAT: a fast spliced aligner with low memory requirements. Nat Methods. 2015;12(4):357-360.

25. Liao Y, Smyth GK, Shi W. The Subread aligner: fast, accurate and scalable read mapping by seedand-vote. Nucleic Acids Res. 2013;41(10):e108.

26. McCarthy DJ, Chen Y, Smyth GK. Differential expression analysis of multifactor RNA-Seq experiments with respect to biological variation. Nucleic Acids Res. 2012;40(10):4288-4297.

27. Ritchie ME, Phipson B, Wu D, Hu Y, Law CW, Shi W, et al. limma powers differential expression analyses for RNA-sequencing and microarray studies. Nucleic Acids Research. 2015;43(7):e47.

28. Chen EY, Tan CM, Kou Y, Duan Q, Wang Z, Meirelles GV, et al. Enrichr: interactive and collaborative HTML5 gene list enrichment analysis tool. BMC Bioinformatics. 2013;14:128.

29. Kuleshov MV, Jones MR, Rouillard AD, Fernandez NF, Duan Q, Wang Z, et al. Enrichr: a comprehensive gene set enrichment analysis web server 2016 update. Nucleic Acids Res. 2016;44(W1): W90-7. 
30. Daniels GH. Follicular Variant of Papillary Thyroid Carcinoma: Hybrid or Mixture?. Thyroid. 2016;26(7):872-874.

31. Agrawal N, Akbani R, Aksoy BA, Ally A, Arachchi H, Asa SL, et al. Integrated genomic characterization of papillary thyroid carcinoma. Cell. 2014;159(3):676-690.

32. Sun C-C, Li S-J, Li G, Hua R-X, Zhou X-H, Li D-J. Long Intergenic Noncoding RNA 00511 Acts as an Oncogene in Non-small-cell Lung Cancer by Binding to EZH2 and Suppressing p57. Mol TherNucleic Acids. 2016;5;e385.

33. Xu S, Kong D, Chen Q, Ping Y, Pang D. Oncogenic long noncoding RNA landscape in breast cancer. Mol Cancer. 2017;16(1):129.

34. Han P, Li JW, Zhang BM, Lv JC, Li YM, Gu XY, et al. The IncRNA CRNDE promotes colorectal cancer cell proliferation and chemoresistance via miR-181a-5p-mediated regulation of Wnt/ $\beta$-catenin signaling. Mol Cancer. 2017;16(1):9.

35. Xie H, Ma B, Gao Q, Zhan H, Liu Y, Chen Z, et al. Long non-coding RNA CRNDE in cancer prognosis: Review and meta-analysis. Clin Chim Acta. 2018;485:262-71.

36. Brodie S, Lee HK, Jiang W, Cazacu S, Xiang C, Poisson LM, et al. The novel long non-coding RNA TALNEC2, regulates tumor cell growth and the stemness and radiation response of glioma stem cells. Oncotarget. 2017;8(19):31785-1801.

37. He H-T, Xu M, Kuang Y, Han X-Y, Wang M-Q, Yang Q. Biomarker and competing endogenous RNA potential of tumor-specific long noncoding RNA in chromophobe renal cell carcinoma. Onco Targets Ther. 2016;9:6399-6406.

38. Wang L, Liu D, Wu X, Zeng Y, Li L, Hou Y, et al. Long non-coding RNA (LncRNA) RMST in triplenegative breast cancer (TNBC): Expression analysis and biological roles research. J Cell Physiol. 2018;233(10):6603-6612.

39. Moretti S, Menicali E, Voce P, Morelli S, Cantarelli S, Sponziello M, et al. Indoleamine 2,3-Dioxygenase 1 (ID01) Is Up-Regulated in Thyroid Carcinoma and Drives the Development of an Immunosuppressant Tumor Microenvironment. J Clin Endocrinol Metab. 2014;99:E832-40.

40. Gonçalves V, Pereira JFS, Jordan P. Signaling Pathways Driving Aberrant Splicing in Cancer Cells. Genes (Basel). 2017;9(1):9.

41. Yea S, Narla G, Zhao X, Garg R, Tal-Kremer S, Hod E, et al. Ras promotes growth by alternative splicing-mediated inactivation of the KLF6 tumor suppressor in hepatocellular carcinoma. Gastroenterology. 2008;134(5):1521-1531.

42. Sastre-Perona A, Riesco-Eizaguirre G, Zaballos MA, Santisteban P. $\beta$-catenin signaling is required for RAS-driven thyroid cancer through PI3K activation. Oncotarget. 2016;7:49435-49449.

43. Ely KA, Bischoff LA, Weiss VL. Wnt Signaling in Thyroid Homeostasis and Carcinogenesis. Genes (Basel). 2018;9(4):E204.

44. Nucera C, Lawler J, Parangi S. BRAF(V600E) and microenvironment in thyroid cancer: a functional link to drive cancer progression. Cancer Res. 2011;71(7): 2417-2422. 
45. Jolly LA, Novitskiy S, Owens P, Massoll N, Cheng N, Fang W, et al. Fibroblast-Mediated Collagen Remodeling Within the Tumor Microenvironment Facilitates Progression of Thyroid Cancers Driven by BrafV600E and Pten Loss. Cancer Res. 2016;76(7):1804-1813.

\section{Additional Material Information}

Additional file 1.xlsx - IncRNA differently expressed in thyroid neoplasms in Microarray dataset;

Additional file 2.xlsx - IncRNA differently expressed in thyroid neoplasms in RNA-Seq PRJEB11591 dataset;

Additional file 3.xlsx - IncRNA differently expressed in thyroid neoplasms in RNA-Seq TCGA dataset;

Additional file 4.pdf - Volcano plots of IncRNA differently expressed in thyroid neoplasms in Microarray, RNA-Seq PRJEB11591 and TCGA dataset;

Additional file 5.txt -IncRNA specific for anaplastic thyroid cancer.

\section{Figures}


A

Low counts in TCGA, $\mathrm{N}=2$

$>$ Not significant in TCGA, $\mathrm{N}=3$

Significant in TCGA , but $\mathrm{FC}<2.0, \mathrm{~N}=15$

Low counts in PRJEB11591, $\mathrm{N}=0$

Not significant in PRJEB11591, N = 1

Significant in PRJEB11591,

but $\mathrm{FC}<2.0, \mathrm{~N}=12$

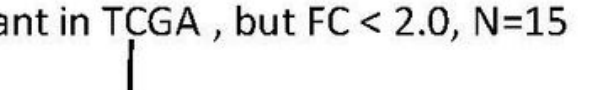




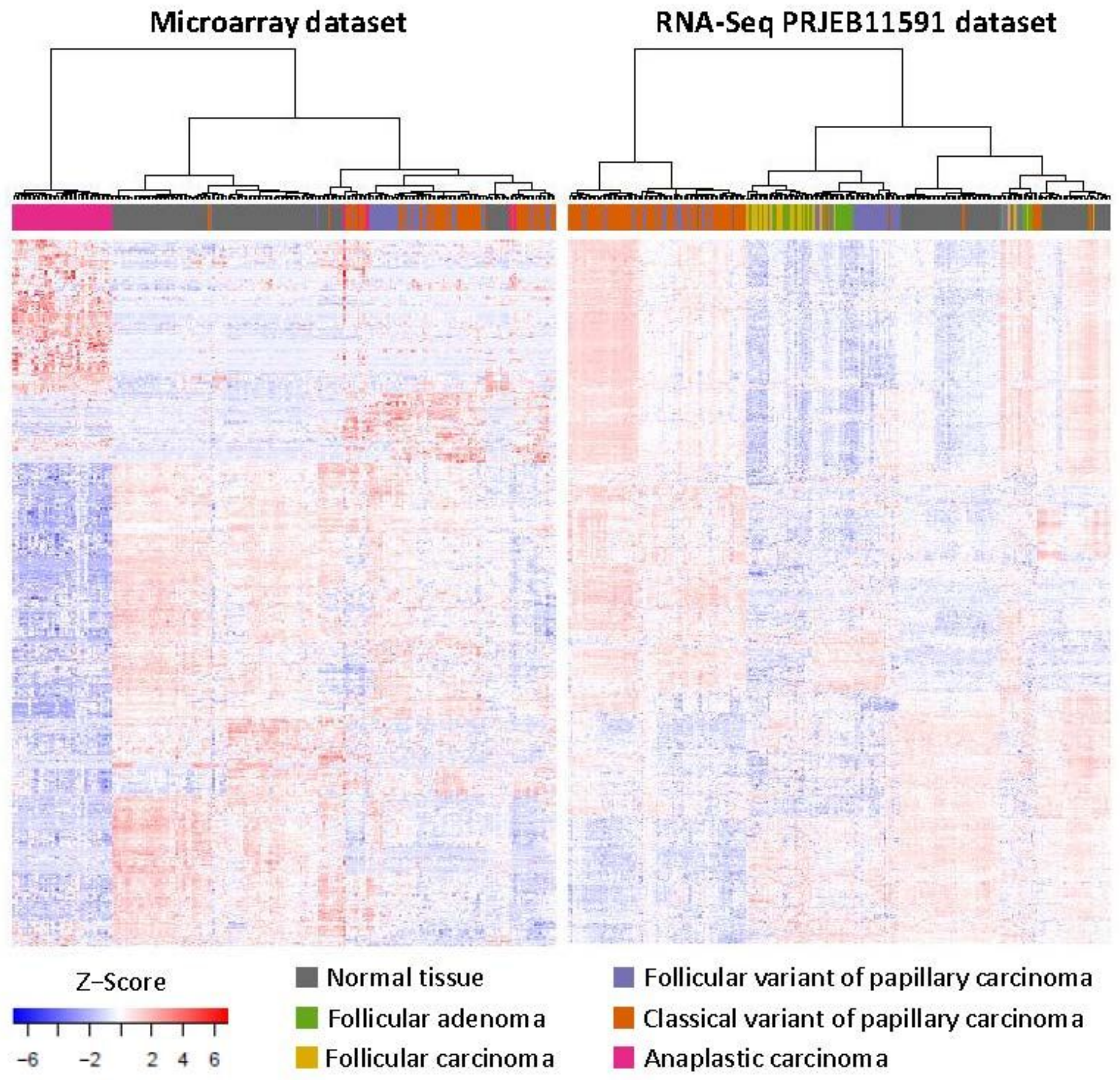

Figure 4

Clustering of FA, FTC, fvPTC, cIPTC and ATC by the expression of IncRNA. A - Microarray dataset; B - RNASeq PRJEB11591 dataset. Genes differently expressed in each histological subtype are included. 


\section{Specific for papillary}

\section{carcinomas}

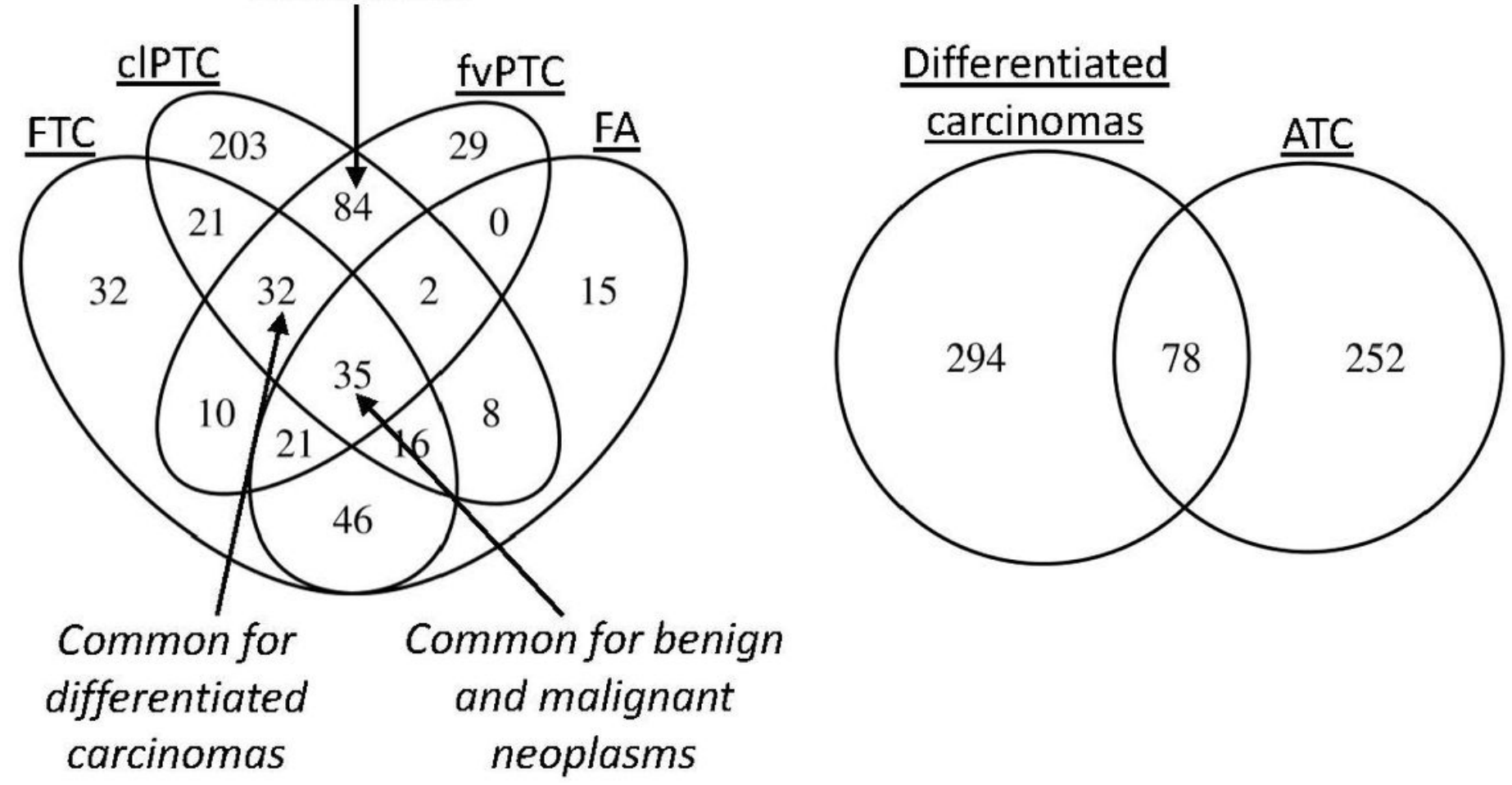

Figure 6

Overlapping of IncRNA landscapes in thyroid neoplasms. A - Overlapping of IncRNA landscapes in differentiated neoplasms - FA, FTC, fVPTC, cIPTC (RNA-Seq PRJEB11591 dataset is used). B Overlapping of IncRNA landscapes in differentiated neoplasms (total list of IncRNA differentially expressed in any of FA, FTC, fvPTC, CIPTC) and ATC. 
Common for benign and malignant neoplasms

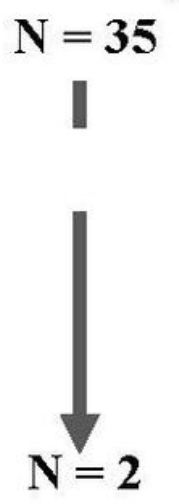

Common for differentiated carcinomas

Validated in fvPTC and clPTC

$$
\mathbf{N}=\mathbf{3 2}
$$

Specific for papillary carcinomas

$$
\mathbf{N}=\mathbf{8 4}
$$

Specific for FTC

$$
\mathbf{N}=\mathbf{3 2}
$$

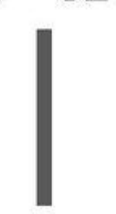

other

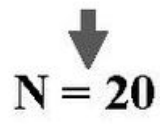

$$
\mathbf{N}=\mathbf{2 0}
$$

Significantly differentially expressed in clPTC \& fvPTC compared to FA

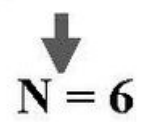
compared to FA \& FTC

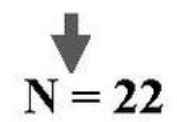

Specific for fvPTC

$$
\mathbf{N}=\mathbf{2 9}
$$

Specific for clPTC

$$
\mathbf{N}=\mathbf{2 0 3}
$$

Validated

Significantly differentially expressed compared to other histological subtypes of differentiated carcinomas

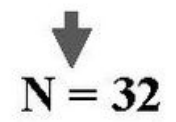

Specific for ATC

$\mathbf{N}=\mathbf{2 5 2}$

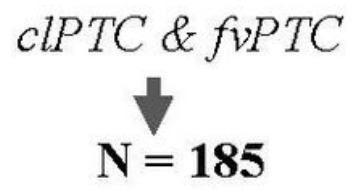

Figure 7

Results of the selection of common and specific IncRNA. 
L1CAM interactions

Specific for FTC

Processing of Capped Intron-Containing Pre-mRNA mRNA Splicing RNA processing Gene Expression mRNA splicing, via spliceosome

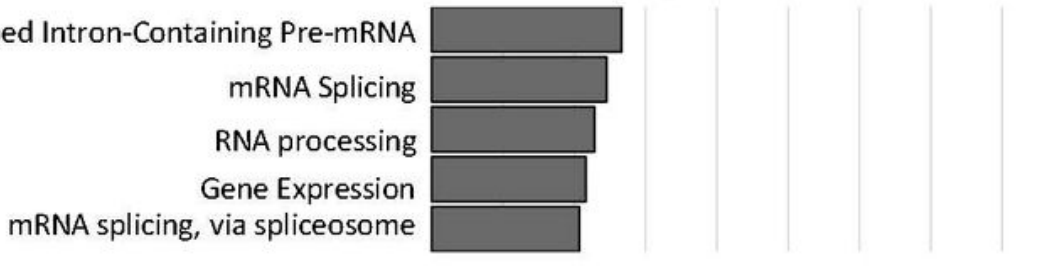

General for papillary carcinomas

Tryptophan metabolism

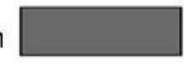

Gene Expression

Beta-catenin independent WNT signaling Autodegradation of $\mathrm{Cdh} 1$ by $\mathrm{Cdh} 1: \mathrm{APC} / \mathrm{C}$

Generic Transcription Pathway Cdc20:Phospho-APC/C mediated degradation of Cyclin A $\mathrm{APC} / \mathrm{C}: \mathrm{Cdh} 1$ mediated degradation of $\mathrm{Cdc} 20$ and other APC/C:Cdh1 APC/C:Cdc20 mediated degradation of Securin APC:Cdc20 mediated degradation of cell cycle proteins prior to satisfation Regulation of activated PAK-2p34 by proteasome mediated degradation

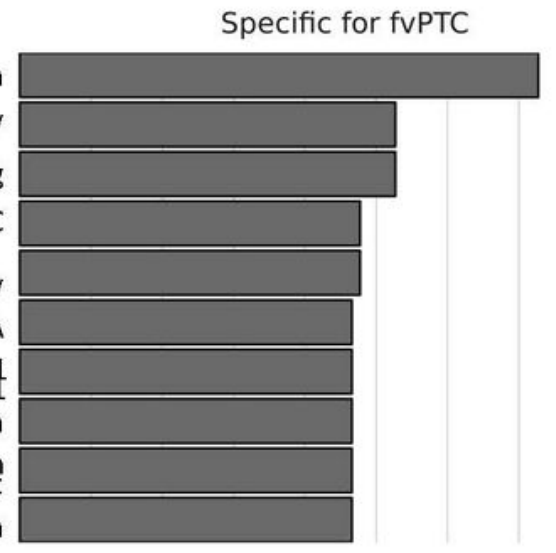

Specific for cIPTC

Extracellular matrix organization

Endoderm formation

Extracellular matrix organization
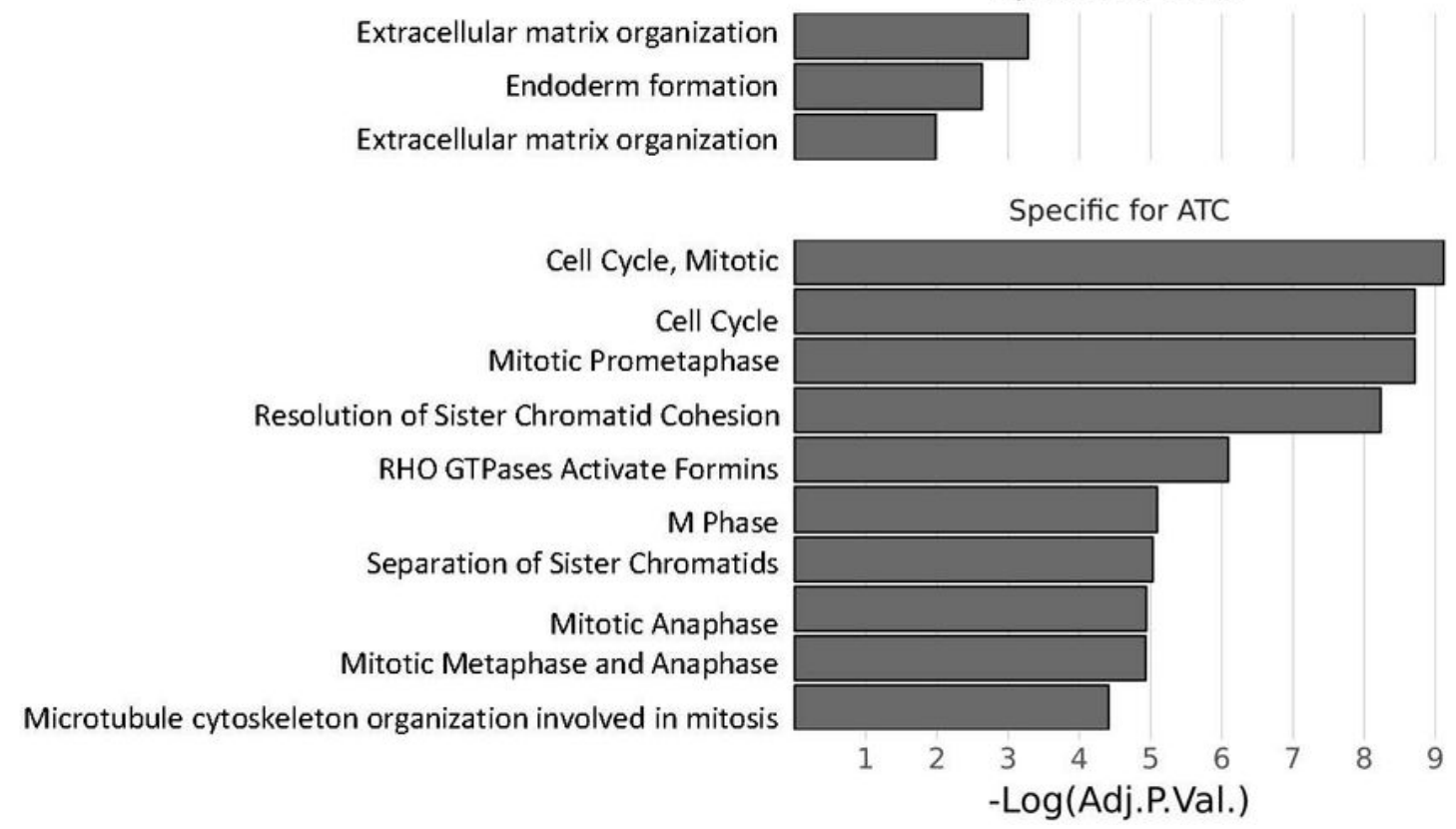

\section{Figure 10}

Putative biological process employing aberrantly expressed IncRNAs in thyroid neoplasms. Enrichment analysis of GO Biological Process, KEGG, and Reactome terms was performed for IncRNA common for thyroid neoplasms, and top 5 IncRNA general for papillary carcinomas, specific for fvPTC, cIPTC, ATC, or FTC. Terms with adjusted $P$ value $<=0.01$ were considered significantly enriched. For fvPTC and ATC top 10 significantly enriched terms are represented. 


\section{Supplementary Files}

This is a list of supplementary files associated with this preprint. Click to download.

- Additionalfile4.pdf

- Additionalfile5.txt

- Additionalfile3.xlsx

- Additionalfile2.xlsx

- Additionalfile1.xIsx

- Additionalfile4.pdf

- Additionalfile2.xlsx

- Additionalfile1.xlsx

- Additionalfile5.txt

- Additionalfile3.xlsx 